\title{
9 \\ Gourous et boîte noire : 50 ans de dérive médiatique
}

> Une corrélation signifie-t-elle un lien de cause à effet?

> Qui était Ancel Keys : un génie ou un homme dans l'erreur?

> Les graisses animales sont-elles mauvaises pour la santé ?

> Que penser des graisses animales?

> Existe-t-il une corrélation entre l'apport lipidique et le cholestérol sanguin, lui-même corrélé à la mortalité coronarienne?

> La découverte des statines, un réel progrès ?

> Les méta-analyses sont-elles le plus puissant des outils?

Selon le Littré, le gourou est un « maître spirituel, maître à penser ».

La boîte noire possède plusieurs définitions : « dispositif enregistrant les données d'un vol », et, dans un sens plus familier et plus proche de notre propos, «caisse secrète d'une association non avouée ». Dans les domaines biologique et pharmacologique, la boîte noire définit une zone non analysée sur le plan scientifique. Elle correspond à l'existence d'une 
corrélation statistiquement significative dans le meilleur des cas, entre cette proposition et l'effet recherché, sans qu'il soit nécessaire de décrypter, démontrer scientifiquement, le mécanisme d'action, la causalité.

Or, pour le discours scientifique, l'existence d'une corrélation n'équivaut, en aucun cas, à l'existence d'une causalité. Certes, il existe des nuances entre une signification à $0,5 \%$ avec $5 \%$ d'erreurs possibles, ou à $0,0001 \%$, mais la certitude, l'élimination des biais possibles, ne peut provenir que de l'élucidation du mécanisme causal et sa reproduction. Faute de quoi, nous restons dans le domaine de la «boîte noire».

\section{Ne pas confondre corrélation et causalité}

Prenons un exemple : si le taux de cancer du sein s'avérait plus élevé parmi les femmes conduisant des voitures rouges, il serait erroné d'en déduire que la couleur de la peinture est responsable des cancers du sein... C'est sans doute seulement le fait que les femmes qui conduisent ce genre de voiture aiment davantage le soleil, le risque, fument plus, etc. Ainsi, une corrélation (nombre de cancers du sein plus élevé chez les conductrices de voitures rouges) ne signifie pas un lien de causalité (la peinture rouge génère des cancers du sein).

Or, comme le disait déjà Julie de Lespinasse dans Le Rêve de d'Alembert de Diderot, "En vérité, il faut être bien circonspect sur ce que l'on assure et sur ce que l'on dit. » Pour franchir allègrement cette difficulté, rien ne vaut l'intervention d'un gourou. Ainsi, boîte noire et gourou sont-ils intimement liés.

\section{MISE EN PLACE DE LA CROISADE CONTRE LES MATIÈRES GRASSES ANIMALES}

Avec la publication d'un article dans la revue Circulation ${ }^{140}$, Ancel Keys entamera la première grande croisade contre la matière grasse

140. Keys A., Taylor H. L., Blackburn H., Brozek J., Anderson J. T., Simonson E. (1963) Coronary heart disease among Minnesota business and professional men followed fifteen years. Circulation, 28, 381-395. 
animale, en faveur de la matière grasse végétale, appuyé par les producteurs de maïs du Minnesota de la "Corn Belt » (la ceinture de production du maiis). Mais revenons quelques années en arrière pour mieux comprendre l'enchaînement de l'histoire.

\section{Des recommandations pour tous}

L'un des premiers travaux d'Ancel Keys, au cours d'une expédition dans les Andes (Chili), fut l'étude des effets comparés de la moyenne et de la haute altitude sur le rythme cardiaque et la pression sanguine pendant seulement 10 jours ("The Physiology of Life at High Altitudes »). Malgré la brièveté du laps de temps étudié, il va, sur la base des résultats obtenus, élaborer des « conseils d'hygiène » à destination des mineurs chiliens travaillant en haute altitude. L'orientation de sa vie future se dessine clairement devant lui.

À partir de ses études, l'homme de sciences se considèrera légitime pour indiquer la conduite à tenir aux chargés des recommandations nutritionnelles, pour montrer au consommateur la supériorité de la régulation dirigée sur la régulation spontanée. Un vertige du pouvoir se dessine.

Il suffit selon lui d'établir les «normes », les « constantes du milieu intérieur ", de les "valider » avec un appareil statistique, puis de les confronter aux résultats obtenus par le biais d'une expérimentation " en boîte noire » de quelques jours (comme les ridicules 10 jours consacrés à l'adaptation à l'altitude !).

Pourtant, dès 1934, le physiologiste britannique Sir Joseph Barcroft (1872-1947) contestait très violemment le diktat de la « fixité du milieu intérieur », énoncé vers 1850 par Claude Bernard ("La fixité du milieu intérieur est la condition de la vie libre»), qui lui semblait "just a little grotesque ${ }^{141}$. Ce type de raisonnement restera étranger à Ancel Keys tout au long de sa carrière.

141. Barcroft H. (1934) Features in the Architecture of Physiological Function. Cambridge: Cambridge University Press. 


\section{Qui était Ancel Keys... avant de devenir le prince des gourous?}

Après un diplôme d'économie et sciences politiques, et une tentative pour devenir gestionnaire, Ancel Keys retourne à l'université de Berkeley et effectue à 25 ans un doctorat d'océanographie et de biologie. Une bourse lui offre l'opportunité de travailler en Europe. Il est accepté au laboratoire du scientifique danois August Krogh (1874-1949), professeur de zoophysiologie à l'université de Copenhague, de 1916 à 1945. Ancel Keys va alors travailler sur des comparaisons entre les poissons d'eau de mer et d'eau douce (différences de régulation du métabolisme du sodium). Sa contribution personnelle est d'avoir utilisé, pour la première fois, une méthodologie statistique pour relier la taille au poids chez les poissons. En réalité, cette méthode avait été décrite par le Belge Auguste Quetelet dès 1880 , reliant le poids à la taille au carré chez l'Homme, sous la forme d'un indice de masse corporelle (le fameux IMC), également appelé indice de Quetelet. Il permettait d'analyser l'influence de récoltes insuffisantes sur la croissance des enfants ${ }^{142}$. Longtemps méconnu, cet indice, très simple, sera utilisé à partir des années 1950 dans les études sur la croissance ou l'adiposité chez l'Homme (indice d'adiposité).

C'est durant les années passées à Copenhague qu'Ancel Keys prend conscience des possibilités qu'ouvre l'analyse statistique des données biologiques, qui représentera l'axe principal de son mode de réflexion. Ces travaux vont lui permettre d'effectuer un doctorat en physiologie à Cambridge en 1936. Il rentre alors aux États-Unis pour occuper un poste au "Laboratoire de la fatigue », à Harvard. En 1937, Ancel Keys est invité à créer, organiser, au sein de l'université du Minnesota un « Laboratoire d'hygiène physiologique » qu'il dirigera jusqu'à sa retraite en 1972. C'est en ce lieu qu'il ouvrira le champ de la « biologie humaine quantitative ", avec un intérêt particulier pour l'étude de l'adaptation aux contraintes environnementales.

142. Quetelet A. (1835) Sur l'homme et le développement de ses facultés, ou Essai de physique sociale. Deux volumes. 


\section{Les rations $K$}

La guerre de 1939-1945 va offrir à Ancel Keys une chance exceptionnelle, après quelques expériences peu démonstratives.

Le 19 novembre 1944, 36 jeunes hommes en pleine santé, objecteurs de conscience, entrent dans une sorte de « camp de concentration expérimental » pour subir une étude de "privation humaine » au laboratoire d'Ancel Keys. Il s'agit d'un équivalent des études en aquarium des poissons d'eau de mer et d'eau douce de sa jeunesse. L'objectif de cette expérimentation est double :

- mieux comprendre les effets d'une semi-privation (1 $800 \mathrm{kcal} /$ jour) dans des conditions de travail définies (3000 cal/jour) et d'activité physique durant 3 mois ;

- préciser les conditions optimales d'un traitement pertinent pour les civils et/ou les militaires ayant subi un type d'agression « analogue » en Europe.

Ancel Keys a pu ainsi observer chez ces " hommes en cage " les conséquences de ce traitement. Les résultats, publiés dans The Biology of The Human Starvation (1950), établiront, aux yeux de certains, sa réputation scientifique. Ils permirent à Ancel Keys d'offrir ses prestations de spécialiste d'hygiène physiologique aux services militaires intéressés, à des industriels, etc. C'est ainsi que la ration $\mathrm{K}$ (environ 800 g pour $3200 \mathrm{kcal} /$ jour), du nom de Keys, fut mise au point pour les troupes aéroportées et se répandit comme un modèle à toute l'armée américaine.

La fin de la guerre dut laisser à Ancel Keys un grand vide. Pourtant, il venait d'ouvrir la voie, à partir de mangeurs autorégulés, savourant la disparition de la faim, à des consommateurs inquiets dont le comportement et la régulation se devaient d'être dirigés, de préférence par des « clercs ", des dogmes et «tabous » : il venait d'introduire le « techno-futurisme » en nutrition. 
"Mangez d'autres graisses " : la naissance de la "diet-heart idea "

Fort opportunément, Ancel Keys se rendit compte que les riches hommes d'affaires du Minnesota présentaient davantage d'accidents cardiaques que leurs employés et, surtout, les Européens qui avaient survécu aux restrictions alimentaires. Cela aboutit à une publication dans Lancet, qui connut très vite une grande renommée : "Prediction of serum-cholesterol responses of man to changes in fats in the diet $»^{143}$. Il existait en physiologie humaine un courant, appelé « Parcimony in Nutrition ", datant de 1909 (Sir J. Crichton-Brown), mais qui ne s'attaquait qu'aux protéines et aux apports glucidiques purement énergétiques. Jusqu'aux années 1940, la vie était facile dans le domaine des lipides. Les matières grasses étaient considérées comme une source d'énergie efficace et pleine d'agréments. La prescription « Mangez moins de graisse », "Eat less fat », n’était pas encore d'actualité.

Mais dans le Minnesota, l'industrie agroalimentaire est puissante et la prescription d'Ancel Keys « Mangez d'autres graisses » (sousentendu des graisses nettement moins dangereuses) laissait entrevoir de tentants horizons. Tous les ingrédients de la mise en place d'un leitmotiv, qui devait se renouveler à l'identique pendant un demisiècle, étaient réunis. Sur la scène médiatique, le diable est incarné par le cholestérol sanguin, les démons par la matière grasse animale (les acides gras saturés), les anges par la matière grasse végétale (les acides gras polyinsaturés). Et le précepte évangélique devait être suivi à la lettre, comme une parole biblique : "Que votre parole soit oui, oui ; non, non; ce qu'on y ajoute vient du malin!» (Matthieu 5.3337). La « diet-heart idea » vient de naître. Qu'importe s'il existe des huiles végétales contenant des acides gras saturés et si des matières grasses animales contiennent des acides gras polyinsaturés, le précepte évangélique ne souffre aucune discussion. Le projet était bâti en soi comme un merveilleux outil de communication.

143. Keys A. et al. (1957) Prediction of serum-cholesterol responses of man to changes in fats in the diet. Lancet, 273 (7003), 959-966. 


\section{La Seven Countries Study}

Aujourd'hui, 55 ans plus tard, les traces qui en restent demeurent très importantes. En 1957, lorsque ce concept fut émis, il ne s'appuyait sur aucune preuve scientifique. Ancel Keys était alors âgé de 53 ans. À la fin des années 1960, au cours d'un congrès à Londres et d'une altercation demeurée célèbre, Sir G. Pickering, grand spécialiste du diabète qui pensait que le problème métabolique était plus complexe, somma Ancel Keys de donner une preuve de ces assertions. Aucune ne fut présentée. C’est seulement le $1^{\mathrm{er}}$ septembre 1963, dans son article intitulé « Coronary heart disease among Minnesota business and professional Men followed 15 years » et publié dans Circulation (voir référence complète plus haut), qu'Ancel Keys livra, à défaut de preuves, des corrélations acceptables. Il s'agissait des résultats de la première étude prospective de la maladie coronarienne. Sur cette base, Ancel Keys put monter, avec ses élèves, la Seven Countries Study ${ }^{144}$, une étude au retentissement considérable menée dans sept pays (Grèce, Italie, Yougoslavie, Finlande, Japon, Pays-Bas, États-Unis), qui s'acheva en 1980. Les livres, monographies et publications scientifiques sur le sujet furent très nombreux, dont l'ouvrage intitulé Seven Countries - A multivariate analysis of death and coronary heart disease (Sept pays - Une analyse multivariée de la mort et de la maladie coronarienne) ${ }^{145}$.

Il faudra attendre plus de 20 ans pour que paraisse, enfin, une critique de cette étude, rédigée par l'épidémiologiste américain Frank B. $\mathrm{Hu}^{146}$ : " in the past too much has been made of ecologic associations between saturated fat and CHD derived from the Seven Countries Study. » (« dans le passé, trop d'importance a été attribuée aux

144. Un site Internet lui est dédié : http://sevencountriesstudy.com/.

145. Keys A., Aravanis C., Blackburn H., Buzina R., Djordjević B. S., Dontas A. S., Fidanza F., Karvonen M. J., Kimura N., Menotti A., Mohacek I., Nedeljković S., Puddu V., Punsar S., Taylor H. L., Van Buchem F. S. P. (1980) Seven Countries. A multivariate analysis of death and coronary heart disease. Cambridge, MA: Harvard University Press, 381 p. ISBN : 0-674-80237-3.

146. Hu F. B. (2004) Book Reviews "Prevention of Coronary Heart Disease ". Kromhout D. et al., eds. Diet, Lifestyle and Risk Factors in the Seven Countries Studies. Norwell, MA : Kluwer Academic Publishers, 2002. Am. J. Clin. Nutr., 79, 168. 
associations écologiques entre acides gras saturés et maladies coronariennes dérivées de l'étude des sept pays »).

\section{Organismes en perpétuelle adaptation}

En 1999, le Britannique J. C. Waterlow, du Département d'épidémiologie et de santé public de la London School of Hygiene \& Tropical Medicine, publiait dans un article intitule "The nature and significance of nutritional adaptation ${ }^{147}$, la synthèse de près d'un siècle de réflexions sur ce sujet, à partir du métabolisme des protéines. Trois formes d'adaptation doivent être distinguées : génétique, physiologique et comportementale. Dans le cadre de l'adaptation physiologique, la notion de temps doit intervenir (rappelons que les études d'A. Keys sur l'adaptation à l'altitude portaient sur une période de 10 jours seulement !). Il faut savoir séparer :

- la régulation à court terme dite homéostatique,

- de la régulation à long terme, très spécifique de l'espèce étudiée,

- de la régulation permettant l'adaptation de l'organisme au plus petit dénominateur commun, ou à la moins efficace des fonctions de l'organisme, à court terme ou définitivement (par exemple, toute diminution de l'efficacité du muscle cardiaque entraîne une diminution de l'oxygénation des tissus, d'où une diminution de l'efficacité d'un certain nombre de fonctions, un ralentissement de la croissance...). Cette adaptation est appelée homéorhésis.

Selon l'expression des physiciens (et notamment du physicien et chimiste belge, d'origine russe, Ilya Prigogine, lauréat du prix Nobel de chimie en 1977), ces adaptations peuvent introduire, surtout à long terme, un changement d'ordre, un nouveau système de régulations. C'est en physiologie fondamentale, puis en pédiatrie, que ces phénomènes ont été les mieux décrits, notamment le redémarrage de la croissance, après un stress majeur, selon une cinétique de « rattrapage » plus rapide que la cinétique de croissance habituelle : c'est le phénomène de « croissance compensatrice endogène » décrit par Schaeffer (1928), désigné également sous le terme de « catch-up » par Prader (1960).

147. Waterlow J. C. (1999) The nature and significance of nutritional adaptation. Eur. J. Clin. Nutr., 53, Suppl. 1, S2-S5. 
L'exemple cité par Waterlow dans le domaine de la nutrition protéique est frappant : " une diminution de la perte azotée obligatoire de seulement $2 \mathrm{mg} /$ jour ne sera pas significative sur le plan statistique sur une période de 1 mois, sur une période de 1 an elle représente une destruction de masse maigre de 1,4 kg environ, principalement au niveau des muscles ». Mais l'inverse est aussi vrai. À terme, l'individu sera-t-il « adapted » à une situation de pénurie (comme estimé par Waterlow ?) ou « depleted », c'està-dire fragilisé (comme décrit par Scrimshaw ?) ? Probablement les deux... La même situation se retrouve dans le domaine lipidique. Ainsi, pour évaluer un apport suffisant en acide linoléique, acide gras indispensable, chez le nourrisson, le meilleur critère est le rapport entre le C20:3 n-9 (reflet de la synthèse endogène totale) et l'acide arachidonique (dérivé de l'acide linoléique). Ce rapport varie de 0,1 à la naissance à 0,04 à 15 jours, à 0,01-0,02 à 115 jours, avec le lait maternel ou le lait de remplacement supplémenté en acide linoléique (Mendy, 1965-1970). Il peut atteindre dans le même temps la valeur de 1 avec un lait de remplacement pauvre en acide linoléique. Dans le cas d'une alimentation parentérale prolongée dépourvue de lipides, il peut finalement atteindre une valeur de 9 ou 10 peu de temps avant une issue fatale.

Dans ces situations extrêmes, la synthèse de lipides endogènes (amenant au C20:3 n-9) explose, le taux d'acide arachidonique (provenant, dans cette seconde phase, davantage des réserves tissulaires que de la transformation de l'acide gras parent linoléique : le rapport acide arachidonique/acide linoléique est en effet supérieur à 1) diminue progressivement... et il peut être totalement nul dans le cas d'une alimentation glucidique parentérale du fait de l'hyperinsulinémie.

Ainsi un flux de synthèse totalement endogène (C20:3n-9) se combine avec une redistribution systémique (acide arachidonique) à partir de réserves tissulaires, d'importance moins grande sur le plan vital, pouvant être remplacée par une synthèse endogène d'acides gras (C20:3 n-9) structurellement proches, via un mécanisme de suppléance endogène. On est loin de la " fixité du milieu intérieur ». Ce mécanisme s'est retrouvé confirmé aux troisième et quatrième âges, avec la description de la lipogenèse de novo et sa pathologie ${ }^{148}$.

148. Wu J. H., Lemaitre R. N., Imamura F., King I. B., Song X., Spiegelman D., Siscovick D. S., Mozaffarian D. (2011) Fatty acids in the de novo lipogenesis pathway and risk of coronary heart disease: The Cardiovascular Health Study. Am. J. Clin. Nutr., 94 (2), 431-438. 


\section{LES ÉQUATIONS PRÉDICTIVES}

La célébrité d'A. Keys commencera en 1957 avec cette équation dite prédictive, établissant une corrélation entre l'apport lipidique et le cholestérol sanguin, lui-même corrélé à la mortalité coronarienne. Cette corrélation est basée sur le rapport $\mathrm{P} / \mathrm{S}$ (acides gras polyinsaturés/saturés) qui, au fil des années, variera de 1 à 2 .

\section{Première équation prédictive (1957) d'A. Keys et al.}

En 1957, pour Ancel Keys, «à moins d'être un poulet ou un lapin », le cholestérol alimentaire ne joue aucun rôle, ce sont les matières grasses alimentaires qui contrôlent le taux de cholestérol sanguin.

\section{Deuxième équation prédictive (1965) d'A. Keys et F. Grande}

La première équation se révèle peu performante. Pour l'améliorer, une relation complémentaire, réintroduisant l'apport en cholestérol alimentaire pour $1000 \mathrm{kcal}$, est avancée. Ce sera la dernière proposition d'Ancel Keys. Mais la mode des équations prédictives est lancée.

\section{Troisième équation prédictive (1965) de Hegsted et al.}

Dans cette troisième équation prédictive, le cholestérol alimentaire est cette fois-ci réintroduit comme tel, en milligramme par jour. Une fois encore, l'efficacité de ces équations prédictives reste très contestée.

\section{Les critiques majeures}

En 1984, le $\mathrm{D}^{\mathrm{r}}$ Edward H. Ahrens, Jr, très grand spécialiste américain des lipides et du cholestérol décédé en 2000, publia un article dressant le bilan de 40 années de surveillance du cholestérol ${ }^{149}$ et proposant ce que l'on appellera le paradoxe d'Ahrens : les régimes riches en acides gras polyinsaturés sont responsables du transfert du cholestérol depuis le plasma sanguin vers les tissus (muscles,

149. Ahrens E. H. Jr (1984) After 40 years of cholesterol-watching. J. Lipid Res., 25 (13), 1442-1449. 
tissu conjonctif, tissu artériel). Une augmentation de ces acides gras polyinsaturés devrait "en toute logique » entraîner une aggravation de la maladie coronarienne ! En 1984, on n'avait pourtant jamais observé une augmentation de cette incidence. À noter que cet « effet » sera mis en évidence, pour des doses très élevées, quelques années plus tard : l'étude des agriculteurs du Var et de la Moselle de Serge Renaud observe ainsi une augmentation de certaines agrégations plaquettaires dangereuses avec des taux élevés, voire très élevés d'acide linoléique.

Enfin, le $\mathrm{D}^{\mathrm{r}}$ Ahrens souligne la nécessité de tenir compte de l'hétérogénéité des populations humaines, dotées de réponses compensatoires d'efficacité variable et du besoin de jonctions pour des études chez l'Homme entre les spécialistes des stérols et les spécialistes de leurs transporteurs (apolipoprotéines, lipoprotéines.)

Trois études épidémiologiques viendront aussi critiquer les équations prédictives, comme indiqué dans nos précédents chapitres:

- une étude australienne de 1978 observera que le lot contrôle (P/S $=0,8)$ obtient de meilleurs résultats que le lot expérimental dont la diète est plus riche en acides gras polyinsaturés $(\mathrm{P} / \mathrm{S}=1,7)$, et ce, de manière significative $(\mathrm{p}<0,01)$;

- l'étude d'Oslo (1986-1987) n'observe de résultat positif en termes de diminution du risque coronarien qu'avec le seul lot contrôle $(\mathrm{P} / \mathrm{S}=0,7)$;

- l'étude Minnesota Coronary Study (1975-1989) comptabilise 248 décès dans le groupe de 4516 personnes consommant $5 \%$ d'acide linoléique en calories $(\mathrm{P} / \mathrm{S}=0,3)$ et 269 morts parmi les 4541 personnes suivant un régime où $14 \%$ de l'énergie est apportée par de l'acide linoléique $(\mathrm{P} / \mathrm{S}=1,6)$. Soit un taux de décès de 55,8 \% dans le groupe supplémenté contre 52,6 \% dans le groupe témoin.

La théorie initiale d'Ancel Keys ne pourra désormais plus être acceptée, sans réflexions critiques. 


\section{L'ANNÉE 1985 : ARRIVÉE SUR LE MARCHÉ DES SYMVASTATINES, INHIBITEURS DE LA SYNTHĖSE DU CHOLESTÉROL}

Le biochimiste et microbiologiste japonais Akira Endō découvre en 1978 le premier inhibiteur de la HMG-CoA réductase (ou premier membre de la famille des statines), alors qu'il s'efforce d'identifier les composés produits par les champignons pour se défendre contre les organismes parasites en inhibant leur synthèse de cholestérol.

Pour la première fois, les mortalités cardiovasculaire et totale baissent. La découverte est majeure, comparable, selon W. C. Roberts ${ }^{150}$, à celle de la péniciline dans le domaine de l'infectiologie: «The underused miracle drugs: the statin drugs are to atherosclerosis what penicillin was to infectious disease. » ("Les médicaments miracles sous-utilisés : les statines sont à l'athérosclérose ce que la pénicilline était à la maladie infectieuse. »)

Nous ne sommes plus dans le "domaine de la boîte noire ": le mécanisme d'action des statines, à savoir l'inhibition de la synthèse du cholestérol, est décrit. C'est la fin d'une époque... avant une rechute.

Pendant ce temps, Ancel Keys prend sa retraite en 1972, à l'âge de 68 ans. Il jouit d'une réputation internationale de quasi-sauveur de l'humanité. Il laisse derrière lui une pléiade de "nageurs de combat ", prêts à défendre ses idées et à mettre toujours en avant de nouvelles équations prédictives, disposées à remplacer, avec le même niveau de certitude déclaré, celles que l'expérience clinique démolit.

On aurait pu imaginer qu'Ancel Keys choisisse de finir ses jours dans le Minnesota, au sein de la civilisation du soja et du maïs, avec le rapport $\mathrm{P} / \mathrm{S}$ comme guide. Il n'en est rien : il se retire en Italie du Sud, près de Naples : la civilisation et l'alimentation méditerranéennes l'ont définitivement converti pour les années qui lui restent à vivre. En 1975, il publia l'ouvrage How to eat well and stay well: The Mediterranean Way. Il décède en 2004, à l'âge de 100 ans.

150. Roberts W. C. (1996) The underused miracle drugs: The statin drugs are to atherosclerosis what penicillin was to infectious disease. Am. J. Cardiol., 78 (3), 377-378. 


\section{LA RECHUTE : LA CONTRE-ATTAQUE DES DISCIPLES}

L'efficacité médiatique d'Ancel Keys ne pouvait tomber en désuétude aussi facilement. D'autant qu'il bénéficie également de toute l'attention politique : la commission sénatoriale de MacGovern publie en 1977 les premières recommandations destinées à enrayer l'épidémie de maladies cardiovasculaires qui touche les États-Unis : moins de graisses saturées, moins de sucres raffinés, davantage de glucides issus des fruits, légumes et graines. Le département américain de l'Agriculture ne pouvait rêver mieux pour soutenir ses productions.

\section{Le retour en force des équations prédictives}

Avec le départ en retraite d'A. Keys, une place de gourou reste à prendre. Au moins trois équipes vont s'y employer avec une même détermination, au travers de la formulation d'équations prédictives successives.

\section{Quatrième équation prédictive (1992 ${ }^{151}$ ) de Mensink et Katan (Pays-Bas, Europe)}

Une quatrième équation prédictive ne tarde pas à voir le jour. Cette fois-ci, les acides gras mono-insaturés viennent s'ajouter aux acides gras polyinsaturés, avec toujours comme facteur dominant maléfique les acides gras saturés. De manière simplifiée, le rapport $\mathrm{P} / \mathrm{S}$ (acides gras polyinsaturés/saturés) devient le rapport I/S (acides gras insaturés/saturés).

Dans cette nouvelle équation, le terme " acides gras insaturés » regroupe les acides oléique, linoléique (oméga-6), alpha-linolénique (oméga-3) et les dérivés hautement insaturés (PUFA) en C20 et C22 des familles oméga-6 et oméga-3. Trois classifications apparaissent : le cholestérol total, le « mauvais cholestérol » (LDL) et le «bon cholestérol »(HDL).

151. Mensink R. P., Katan M. B. (1992) Effect of dietary fatty acids on serum lipids and lipoproteins. A meta-analysis of 27 trials. Arterioscler. Thromb., 12 (8), 911-919. 
Mais il est observé que les acides gras saturés ont une action positive, bénéfique, sur le "bon cholestérol ». Un véritable paradoxe, difficilement soluble, dans une équation simple.

\section{Cinquième équation prédictive $\left(1993^{152}\right)$ de Hegsted et al. (États-Unis)}

Dans cette cinquième tentative de prédire, via une simple équation, la complexité de la régulation des lipides, le rapport $\mathrm{P} / \mathrm{S}$ existe toujours, mais une influence du cholestérol alimentaire est ajoutée. L'influence sur le « mauvais cholestérol » (cholestérol LDL) prend en compte le cholestérol alimentaire. Et ce n'est que pour le «bon cholestérol » que l'influence des acides gras mono-insaturés est prise en compte, associée à celle du cholestérol alimentaire.

\section{Sixième équation prédictive $\left(1995^{153}\right)$ de Yu et al. (États-Unis)}

Dans cette sixième tentative, le facteur $S$ est fragmenté en $\mathrm{C} 12: 0+$ C16:0 + C18:0. Acides gras mono-insaturés et polyinsaturés s'additionnent avec un coefficient différent. Globalement, c'est le rapport acides gras insaturés sur saturés qui devient le rapport dominant.

$\mathrm{Au}$ niveau du cholestérol LDL, la nouvelle classification, acides gras insaturés/saturés, est prise en compte. Il en va de même pour le «bon cholestérol » (HDL) : une formule de plus en plus complexe associe de façon positive, bénéfique, le C12:0, le C14:0 et le C16:0 et, de façon négative, le C18:0 ainsi que les acides gras mono-insaturés et ceux polyinsaturés.

La situation de ces équations prédictives devient quelque peu « grotesque ». En 1999, durant le congrès de l'International Society

152. Hegsted D. M., Ausman L. M., Johnson J. A., Dallal G. E. (1993) Dietary fat and serum lipids: An evaluation of the experimental data. Am. J. Clin. Nutr., 57 (6), 875-883.

153. Yu S., Derr J., Etherton T. D., Kris-Etherton P. M. (1995) Plasma cholesterolpredictive equations demonstrate that stearic acid is neutral and monounsaturated fatty acids are hypocholesterolemic. Am. J. Clin. Nutr., 61 (5), 1129-1139. 
for Fat Research qui se tient à Brighton, le vice-président d'Unilever publiait "An Alien Called Consumer», souhaitant un retour à l'année 1957 et au bon vieux rapport $\mathrm{P} / \mathrm{S}$ : « Maybe it was too simple» («Peutêtre que c'était trop simple»), " mais nous avons surchargé le consommateur, avec des débats sur le rapport $P / S$ idéal, sur le rapport n-3/n-6, sur le maximum de $10 \%$ d'énergie provenant des polyinsaturés... ».

En 1994, la Scandinavian Survival Study, étude portant sur les statines qui inhibent la synthèse du cholestérol, met en évidence une baisse de $25 \%$ du cholestérol total et du cholestérol LDL. On est loin des petits $5 \%$ revendiqués par les études épidémiologiques et les équations prédictives.

\section{LE RENVERSEMENT DES POSITIONS DÉFINITIVES SUR LES ACIDES GRAS SATURÉS}

\section{Le retour à la nuance}

Les années 2000 semblent marquer le retour à la nuance. En 2001, $\mathrm{V}$. Krog révèle le rôle positif de l'indice de saturation des phospholipides des membranes.

En avril 2002, le spécialiste américain W. E. M. Lands, reprenant un article majeur publié ailleurs, écrit ces lignes (Inform, 13, 346): "It was not clear at the time (or even today!) what the saturated fat might actually be doing to harm health» (« La raison pour laquelle les matières grasses saturées peuvent actuellement poser des problèmes de santé n'est pas claire en ce moment (ni même à ce jour !) »).

En 2004, le château de cartes construit par Ancel Keys s'écroule, à cause de l'épidémiologiste américain Frank $\mathrm{B}$. $\mathrm{Hu}^{154}$ : « in the past too much has been made of ecologic associations between saturated fat and CHD derived from the Seven Countries Study» (« dans le passé,

154. Hu F. B. (2004) Book Reviews « Prevention of Coronary Heart Disease ». Daan Kromhout et al., eds. Diet, Lifestyle and Risk Factors in the Seven Countries Studies. Norwell, MA : Kluwer Academic Publishers, 2002. Am. J. Clin. Nutr., 79, 168. 
trop d'importance a été attribuée aux associations écologiques entre acides gras saturés et maladies coronariennes dérivées de l'étude des sept pays »).

Cette prise de conscience fut confirmée, dans le même temps, par le $\mathrm{Pr}^{\mathrm{r}}$ Jose Ordovas, disciple de F. Grande, un collaborateur d'Ancel Keys depuis 1955.

Mais la vague des "interventionnistes ", adeptes de la boîte noire, n'est pas retombée pour autant. Trop d'oreilles avides de certitudes que l'on peut claironner, et que viendra conforter le principe de précaution, leur étaient attentives depuis très longtemps : celles des spécialistes du marketing agroalimentaire, celles des organismes publics chargés des recommandations, celles des politiques soucieux de faire la police « du Bien ou du Mal ». Qu'importe les réserves, même celles de mathématiciens comme Yoav Benjamini et Yosef Hochberg ${ }^{155}$ sur les analyses multiples et la nécessité de contrôles rigoureux... La tentation restait très grande.

\section{L'arrivée des méta-analyses et du big data}

L'arrivée des méta-analyses et du big data va permettre de relancer à nouveau le débat.

Selon l'expression de W. E. M. Lands, certains épidémiologistes se sont eux aussi enfermés dans une «silo mentality», devenant hermétiques à l'évolution, scientifique ou autre, du monde extérieur.

Mais la réponse est publiée cette fois-ci en même temps que la relance du débat.

À l'article "Associations between food and beverage groups and major diet-related chronic diseases: An exhaustive review of pooled/ meta-analyses and systematic reviews ${ }^{156} »$ publié par les Français A. Fardet et Y. Boirie répond l'éditorial «Big data and systematic

155. Yoav B., Hochberg Y. (1995)Controlling the False Discovery Rate: A Practical and Powerful Approach to Multiple Testing. Journal of the Royal Statistical Society. Series B (Methodological), 57, 1, 289-300.

156. Nutr. Rev. (2014), 72 (12), 741-762. 
reviews in nutritional epidemiolology " ${ }^{157}$ cosigné par les Américains A. Satija et F. B. Hu.

Pour ces derniers, combiner dans les méta-analyses les informations issues de différentes études se heurte vite à un mur : il est difficile de comparer les effets obtenus avec des types de consommations multiples, des études de dimensions différentes, d'hétérogénéité sousjacente variable, de qualité méthodologique fluctuante, etc. Sur le plan scientifique, l'objectif poursuivi serait presque impossible. La " méta-épidémiologie » serait finalement moins efficace que l'étude sérieuse des essais cliniques. Au mieux, nous sommes au commencement de ce qui devrait devenir une méthodologie rigoureuse conduisant à des revues d'évidence en épidémiologie nutritionnelle et à une étape vers la solution du challenge de la synthèse et la compréhension du big data. 


\section{» À retenir}

L'existence d'une corrélation n'équivaut pas à un lien de causalité.

Une certitude scientifique ne pourra jamais provenir que de la découverte, de la reconstitution d'un mécanisme causal.

De nombreux " gourous » ont proposé des relations non démontrées, n'ayant pas pris le temps de décrypter la "boite noire » des mécanismes sous-jacents. Le plus célèbre d'entre eux fut Ancel Keys, qui mena une croisade médiatique contre les graisses animales saturées qu'il diabolisa. La Seven Countries Study, publiée en 1963, fut une étude au retentissement considérable et qui, pourtant, souffrait de sévères limites. Elle marqua le début d'une tentation : celle de proposer des équations prédictives établissant une corrélation entre l'apport lipidique et le cholestérol sanguin, lui-même corrélé à la mortalité coronarienne.

La découverte des statines en 1978, non seulement très efficaces (baisse de $25 \%$ du cholestérol total et du cholestérol LDL) mais aux mécanismes d'action décryptés (pas de boîte noire), marquera la fin de cette période de statistiques prédictives.

À partir des années 2000 , le temps de la nuance semble arrivé : c'est la fin de la diabolisation des graisses saturées et la remise en cause des associations dérivées de l'étude des Seven Countries d'Ancel Keys.

La tentation de prédire, de trouver des corrélations simples et des messages manichéens (les "bons » et les " méchants ») reste grande: à ce titre, la mode des méta-analyses et du big data pourrait venir remplacer les équations prédictives. 